Recebido em 05/2013. Aceito para publicação em 05/2014.

\title{
AVALIAÇÃO DA ESTRUTURA FISICA DE UMA UNIDADE DE ALIMENTAÇÃO E NUTRIÇÃO
}

\section{EVALUATION OF THE PHYSICAL STRUCTURE OF A FOOD AND NUTRITION UNIT}

\author{
Noeli Fatima Pohren ${ }^{1}$ \\ Gislaine Angela Martinazzo ${ }^{1}$ \\ Maielenn Biazoli dos Anjos ${ }^{2}$ \\ Mirian Cozer ${ }^{3}$
}

\begin{abstract}
Resumo: Unidade de Alimentação e Nutrição (UAN) é um estabelecimento responsável pela produção de refeições, cuja função é fornecer dieta balanceada, ou seja, em quantidade suficiente, com qualidade completa e adequada às características e hábitos alimentares dos usuários, bem como, segura do ponto de vista higiênico. Este estudo caracteriza-se por uma pesquisa metodológica, descritiva que buscou avaliar as condições da estrutura física de uma UAN, localizada em uma cidade do sudoeste do Paraná, usando como critério a $R D C$ no 275, de 21 de Outubro de 2002, para determinar se as condições estruturais encontram-se em situações propícias à obtenção de alimentos seguros. As opções de respostas para o preenchimento da lista de verificação foram: para conforme (C), para os itens não conforme (NC), para os itens não atendidos, e não se aplica (NA), aos itens observados nos estabelecimentos avaliados. Após a coleta de dados, o estabelecimento recebeu a classificação, que se refere a uma pontuação de 0 a $50 \%$ de atendimento dos itens, enquadrando-se ao grupo 3 com percentual de $39 \%$ dos itens observados em conformidade. Diante dos resultados obtidos, pode-se perceber algumas falhas quanto à estrutura física da UAN. Percebendo-se a necessidade de correções para garantia da qualidade dos alimentos, conforme a padronização correta quanto à estrutura física de uma UAN, concluindo-se que a unidade encontra-se em condições insatisfatórias, indicando mudanças que visem à melhoria da unidade, como a implantação de sistemas de qualidade na estrutura física da UAN.
\end{abstract}

Palavras-chave: Unidade de Alimentação e Nutrição; estrutura física; lista de verificação.

Abstract: A Food and Nutrition Unit (FNU) is an establishment responsible for the production of meals, which aims to provide a balanced diet, and food that is of sufficient quantity, good quality, and appropriate for the characteristics and habits of its users, as well as safe from the point of view of hygiene. This methodological study used descriptive research to evaluate the conditions of the physical structure of a FNU, in a city located in southwestern Paraná. We used the RDC n- 275 criteria of 21 October 2002, to determine whether the structural conditions are conducive to obtain safe food. The response options for completing the checklist were for items in compliance (C), for items not compliant (NC), for items missed and not applicable (NA) for the items noted in the evaluated establishments. After data collection, the establishment was classified, which refers to a score from 0 to $50 \%$ of the items covered, with group 3 obtaining a percentage of $39 \%$ of the items noted accordingly. The results obtained found some problems in the physical structure of FNU. There was need for correction to ensure the quality of food, to conform to the correct standard for physical structure of an FNU, concluding that the unit in poor condition requires changes to improve the unit, to implement quality systems in the physical structure of the FNU.

Keywords: Food and Nutrition Unit, physical structure; checklist.

1 Graduanda em Nutrição - Universidade Paranaense - Unipar, Brasil. E-mails: noelipohren@hotmail.com; gi_martinazzo@hotmail.com.

2 Especializada em Nutrição Humana - Unipar e Docente da Unipar. E-mail: maielenn@unipar.br.

${ }^{3}$ Especializada em Nutrição Clínica - Faculdade Assis Gurgacz - FAG, Brasil e em Saúde Pública - Universidade Estadual do Oeste do Paraná - Unioeste, Brasil e Docente da Unipar. E-mail: miriancozer@yahoo.com.br. 


\section{INTRODUÇÃO}

Segundo Teixeira et al. (2004), as Unidades de Alimentação e Nutrição (UAN) são unidades de trabalho ou órgão de uma empresa, que tem por finalidade desempenhar atividades relacionadas à alimentação e nutrição que objetiva garantir instalações adequadas e funcionais, assegurando a operacionalização dentro das mais rígidas normas técnicas e de higiene, bem como a qualidade da produção de serviço prestado aos comensais, o planejamento físico adequado, aliado à escolha certa dos equipamentos e o número correto de funcionários, influenciando, diretamente, na qualidade do serviço a ser prestado e nas condições higiênico-sanitárias (MEZOMO, 2004; GUIMARÃES, 2006).

O controle das condições higiênico-sanitárias, nos locais em que os alimentos são manipulados, constitui um ponto crítico de controle, uma vez que contaminações de diferentes fontes podem ser introduzidas nas diversas etapas do preparo do alimento (SÃO JOSE; PINHEIRO-SANT'ANA, 2008). Fato este que leva os governantes a formularem leis para o controle das condições higiênico-sanitárias em relação a todo processo produtivo dos alimentos, que vai, desde a matéria-prima, até o produto final, que é o prato do consumidor (NASCIMENTO; SILVA, 2007).

As Unidades de Alimentação e Nutrição possuem estrutura organizacional simples, mas se tornam complexas, dependendo do tipo e quantidade de refeições produzidas e o tipo de gerenciamento e contrato (COLARES; FREITAS, 2007). Pertencem ao setor de alimentação coletiva e possuem um compromisso com a saúde, pois oferecem aos comensais uma alimentação equilibrada, respeitando as Leis da Alimentação, como a Quantidade, Qualidade, Harmonia e Adequação. Elas devem ser alinhadas com as exigências das normas da Vigilância Sanitária para ofertar aos comensais alimentos sem causar riscos ou danos à sua saúde. Para atingir tais exigências, é necessário um nutricionista como responsável técnico (SANT'ANNA; GOMES, 2010).

Segundo a FAOMHO (UNITED NATIONS ORGANIZATION, 2003), segurança de alimentos é a garantia de que o consumo de um determinado alimento não the cause danos, quando preparado ou consumido de acordo com o seu uso intencional. O alimento deve estar livre de contaminantes químicos, físicos e microbiológicos. O consumidor, por sua vez, deve ser capaz de exigir a qualidade microbiológica, sensorial e nutricional do alimento que adquire.

O tipo de serviço de autogestão significa serviço próprio, em que a empresa beneficiária assume toda a responsabilidade pela elaboração das refeições, desde a contratação de pessoal até a distribuição aos usuários (BRASIL, 1997).

Neste estudo, foi utilizada a lista de verificação, preconizada pela ANVISA, Agência Nacional de Vigilância Sanitária, tendo a Resolução da Diretoria Colegiada (RDC) nำ275, de 21 de Outubro de 2002, como um dos instrumentos para o acompanhamento da análise da estrutura física da Unidade de Alimentação e Nutrição.

Tem-se como objetivo principal, deste estudo, analisar a estrutura física de uma Unidade 
de Alimentação e Nutrição (UAN), localizada em um município do sudoeste do Paraná, por intermédio da aplicação de uma lista de verificação, adaptada da RDC $n=275$, de 21 de Outubro de 2002.

\section{METODOLOGIA}

O presente estudo caracteriza-se por ser metodológico, descritivo, levando, como base de seu delineamento, as questões ou problemas específicos, utilizando questionários e entrevistas (DALFOVO; LANA; SILVEIRA, 2008). Para a coleta de dados, utilizou-se, como método, a pesquisa quantitativa aliada à qualitativa, pois os dados obtidos foram analisados, indutivamente, tendo a interpretação e a atribuição de significados (RODRIGUES, 2007).

A pesquisa foi desenvolvida em uma Unidade de Alimentação e Nutrição (UAN), de um município do Sudoeste do Paraná, durante o primeiro semestre de 2013 , tendo como objetivo verificar as condições higiênico-sanitárias do local.

Para a avaliação, utilizou-se uma lista de verificação, adaptada da RDC n 275 , de 21 de outubro de 2002, sendo utilizados, para classificação do estabelecimento, os pontos de não conformidade, contendo 18 itens, divididos em 1 grupo, sendo eles edificação e instalações.

Para a compilação dos dados, foi utilizado o método de porcentagens para a classificação dos itens, conforme, não conforme, e não se aplica. Ao final das avaliações, os resultados foram classificados como, Grupo 1 de 76 a 100\% de atendimento dos itens; Grupo 2, de 51 a $75 \%$ de atendimento dos itens; e Grupo 3 de 0 a $50 \%$ de atendimento dos itens, baseados na RDC no 275 .

\section{RESULTADOS E DISCUSSÕES}

De acordo com a aplicação da lista de verificação, adaptada da RDC no 275/2002, identificou-se um percentual de 50\% para os itens não conformes e 39\% para os itens em conformidade e $11 \%$ dos itens, propostos pelo guia de verificação, não se aplica. Com base nesses resultados, observou-se que a maioria dos itens estava fora do que é preconizado pela legislação vigente, sendo que o estabelecimento classificou-se no grupo 03 de adequação dos itens, conforme mostra Gráfico 1 e Tabela 1. 
Gráfico 1 - Distribuição em percentual dos itens conformes, não conformes e não se aplica.

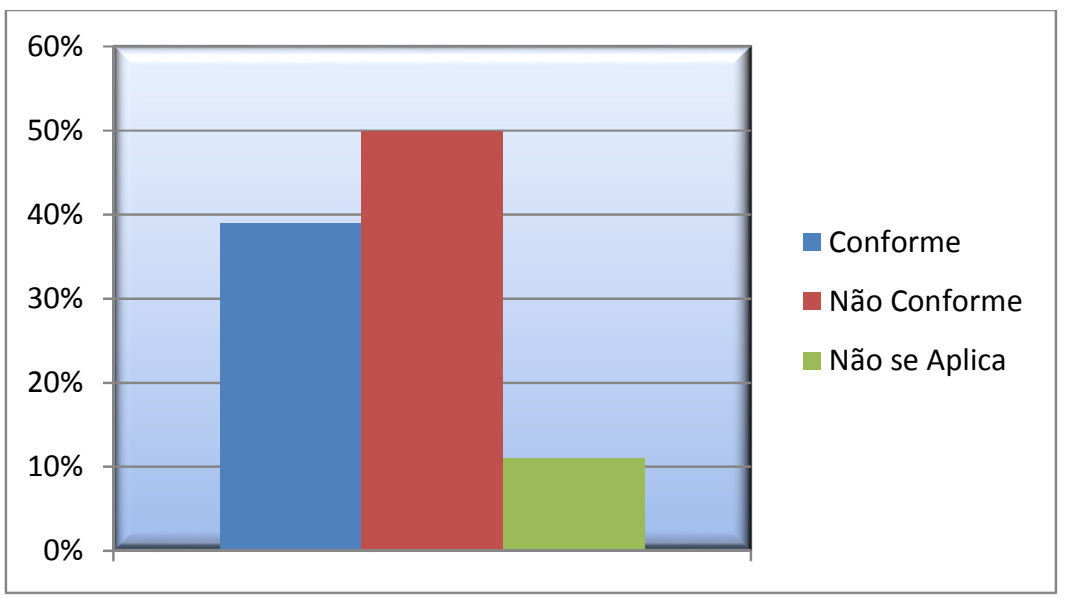

Tabela 1 - Distribuição dos dados de acordo com os grupos analisados, nas unidades avaliadas

\begin{tabular}{lccc}
\multicolumn{1}{c}{ Grupos } & Conforme & Não conforme & Não se aplica \\
\hline 1 - Edificações e instalações & 23 & 51 & 5 \\
2 - Equipamentos moveis e utensílios & 10 & 11 & 0 \\
3 - Manipuladores & 8 & 6 & 0 \\
4 - Produção e transporte do alimento & 20 & 10 & 3 \\
5 - Documentação & 2 & 5 & 11 \\
TOTAL DE ITENS & 63 & 83 & 19 \\
\hline
\end{tabular}

Para os tópicos encontrados em não conformes, de acordo com a RDC n 275/2002, encontrou-se a área externa com focos de insalubridade, de objetos em desuso ou estranhos ao ambiente, de vetores e outros animais no pátio e vizinhança, de focos de poeira, de acúmulo de lixo nas imediações, de água estagnada. Em um estudo realizado por Rodrigues e Martins (2008), em três restaurantes de Cascavel, foram encontrados, em um dos três restaurantes avaliados, dados semelhantes, apresentando objetos em desuso, focos de proliferações de insetos e roedores na área externa da unidade.

A unidade de alimentação e nutrição também apresentou não conformidade em relação ao piso, o qual é de cor cinza escuro, apresentando rachaduras, em alguns lugares, faltando parte do rejunte, podendo ser fonte de contaminação para os alimentos. Encontram-se ralos, em meio à cozinha, que não possuem o enclinamento necessário para o escoamento dos restos de alimentos, ficando ali depositados, gerando contaminação no local. Em um estudo realizado por Liberto, Landim e Costa (2009), observou-se que existiam alguns azulejos no piso, trincados e quebrados, necessitando substituição.

Outra não conformidade, encontrada na UAN, está relacionada ao teto, o qual apresenta mofos, bolores, rachaduras, com tubulações expostas e, até mesmo, em alguns pontos, descascamento, dando a perceber a ausência de manutenção da estrutura. Pimentel (2006) encontrou resultados semelhantes em seu estudo, o teto apresentou mofos e 
trincos, com tubulações expostas, o que mascara a cor clara do teto, dificultando a higienização, com riscos de vazamentos, podendo haver contaminação dos alimentos e, em um estudo realizado por Souza (2006), em uma unidade de alimentação e nutrição hoteleira, encontrou-se esse item em boas condições de limpeza e conservação.

Verificou-se, ainda, em relação à edificação, que as aberturas, como portas e janelas, não possuem fechamento automático, não possuem barreiras para impedir a entrada de pragas, vetores e outros animais. Em estudos realizados em unidade de alimentação e nutrição de uma Organização Militar da cidade de Belém, no Pará, encontraram resultados semelhantes, pois não possuía barreiras contra a entrada de vetores e pragas urbanas (VIDAL et al., 2011; SOUSA et al., 2009). Em outro estudo, realizado por Oliveira (2004), as portas possuíam fechamento automático (mola), pisos e paredes são adequados e apresentam satisfatório estado de conservação, provenientes de iluminação natural e artificial, a ventilação é conseguida por meio de janelas teladas, localizadas na parte superior das paredes, com comunicação direta com o exterior.

As instalações sanitárias e vestiários para os manipuladores encontraram- se separadas por sexo, as torneiras não são com acionamento automático e sim manual, as portas são com fechamento manual, pisos e paredes com trincos e com mofos, iluminação somente elétrica, as lixeiras são com tampas e com acionamento manual, vestiários sem divisórias individuais para cada funcionário, ausência de armários, não possui instalação sanitária para visitantes, os quais têm que utilizar o local dos funcionários. Em um estudo realizado por Machado (2009), em Serviços de alimentação de organizações não governamentais de Toledo, no Paraná, encontraram-se resultados diferentes, em que as instalações sanitárias eram separadas para manipuladores de alimentos e para visitantes.

A iluminação é artificial, pois possui algumas janelas que dão acesso ao setor de estoque, mas que não fornecem luz ao suficiente local de produção, as luminárias não possuem proteção contra quebras, não ocorre circulação de ar e nem ventilação, apenas contém exaustores para minimizar o calor na área. Em um estudo realizado por Guimarães (2006), e m um restaurante de Brasília, encontraram-se fatos semelhantes, sendo que a área de produção possui apenas 01 (uma) janela, com 2,5m de comprimento por $1 \mathrm{~m}$ de largura, constituída de ferro e tela de arame milimetrada, as telas são removíveis, facilitando a limpeza, o número de janelas é insuficiente, aliado ao fato do local se situar no subsolo, faz com que, praticamente, não haja a presença de luz natural.

\section{CONCLUSÃO}

De acordo com a lista de verificações aplicada, a UAN está enquadrada no Grupo 03, de 0 a $50 \%$ de atendimento dos itens, com um percentual de $39 \%$ para os itens não conforme, não estando dentro das conformidades do que preconiza a RDC no 275, de 21 de outubro de 2002. Diante disso, a realização de uma análise da estrutura física e funcional subsidiam o entendimento da importância do serviço e sinalizam as mudanças que visem 
à melhoria do setor, como a implantação de sistemas de qualidade na estrutura física da UAN.

\section{REFERÊNCIAS}

BRASIL. Ministério do Trabalho e Emprego. Programa de Alimentação do Trabalhador - PAT. Portaria MTB no 87, de 28 de janeiro de 1997. DOU, 29/01/1997. Disponível em: < http://portal.mte.gov.br/pat/portarias.htm>. Acesso em: 10 maio 2013.

COLARES, L. G. T.; FREITAS, C. M. Processo de trabalho e saúde de trabalhadores de uma unidade de alimentação e nutrição: entre a prescrição e o real do trabalho. Cad. Saúde Pública, Rio de Janeiro, v. 23, n. 12. pp. 3011-3020, dez. 2007. Disponível em: <http://www.scielo.br/pdf/csp/v23n12/21>. Acesso em: 23 abr. 2013.

DALFOVO, M. S.; LANA, R. A.; SILVEIRA, A. Métodos quantitativos e qualitativos: um resgate teórico. Revista Interdisciplinar Científica Aplicada, Blumenau, SC, v. 2, n. 4, pp. 01-13, 2008.

GUIMARÃES, I. A. Análise da estrutura física e funcional de um restaurante em Brasília. 2006. 65f. Trabalho de Conclusão de Curso (Especialização em Gastronomia como Empreendimento), Universidade de Brasília, Brasília, DF, 2006.

LIBERATO, K. B. L.; LANDIM, M. C.; COSTA, E. A. Estrutura física da área de produção de uma unidade de alimentação e nutrição (UAN) localizada em Fortaleza, CE. In: CONFRESSO BRASILEIRO DE ECONOMIA DOMÉSTICA. 20, 2009, Fortaleza. Trabalhos... Fortaleza, 2009. Disponível em: <http://www.xxcbed.ufc.br/arqs/gt6/gt6 50.pdf>. Acesso em: 27 mar. 2013.

MACHADO, M. R. M. Avaliação das condições de higiene manipulação de alimentos do restaurante universitário da Universidade Estadual de Londrina. 2009, 15f. Trabalho de Conclusão de Curso (Especialização em Gestão Pública) - Instituto Superior de Educação do Paraná, Londrina, PR, p. 15, 2009.

MEZOMO, I. Os serviços de alimentação: planejamento e administração. São Paulo: Terra, 2004.

NASCIMENTO, K. O.; SILVA, E. B. Avaliação das condições higiênico-sanitárias de panificadoras em Volta Redonda, Rio de Janeiro. Revista Nutrição em Pauta, São Paulo, v. 21 , n. 157 , p. $61-64,2007$.

OLIVEIRA, A. M. Boas práticas de fabricação em uma unidade de alimentação do Distrito Federal. 2004, 77f. Trabalho de Conclusão de Curso (Especialização em Qualidade dos Alimentos) - Universidade de Brasília. Brasília, 2004.

PIMENTEL, R. C. Análise da estrutura física de uma unidade de alimentação e nutrição hospitalar do Distrito Federal. Trabalho de Conclusão de Curso (Especialização em Qualidade de Alimentos) - Universidade de Brasília, Centro de Excelência em Turismo, Brasília, 2006.

RODRIGUES, S.; MARTINS, A. H. Avaliação da estrutura física em unidades de 
alimentação e nutrição da cidade de Cascavel - PR. 2008. 13f. Trabalho de Conclusão de Curso (Nutrição) - FAG, Cascavel, PR, 2008.

RODRIGUES, W. C. Metodologia científica. Paracambi, RJ: FAETEC/IST, 2007. Disponível em:

$<$ http://professor.ucg.br/sitedocente/admin/

arquivosupload/3922/material/willian\%20costa\%20rodrigues metodologia cientifica.pdf>.

Acesso em: 08 abr. 2013.

SANT'ANNA, R. F. D.; GOMES, R. F. M. Avaliação das condições higiênicas sanitárias em uma unidade de alimentação e nutrição em Brasília, DF. 2010. Disponível em: $<$ http://www.unisc.br/portal/upload/com arquivo/metodos quantitativos e qualitativos um $r$ esgate teorico.pdf>. Acesso em: 12 maio 2013.

SÃO JOSÉ, J. F. B.; PINHEIRO-SANT'ANA, H. M. Avaliação das boas práticas de manipulação em unidade de alimentação escolar. Nutr.: Rev. Soc. Bras. Alim. Nutr. São Paulo, v. 33, n. 3, p. 123-138, dez. 2008. Disponível em: <http://revistanutrire.org.br/files/v33n3/v33n3a10.pdf >. Acesso em: 27 mar. 2013.

SOUSA, C. L. et al. Diagnóstico das condições higiênico sanitárias e microbiológicas de empresa fornecedora de comidas congeladas light na cidade de Belém - PA. Alim. Nutr., Araraquara, v. 20, n. 3, p. 375-381, 2009.

SOUZA, L. H. L. A manipulação inadequada dos alimentos: fator de contaminação. Revista Higiene Alimentar, São Paulo, v. 20, n. 146, p. 32-39, 2006.

TEIXEIRA, S. et al. Administração aplicada às unidades de alimentação e nutrição. São Paulo: Atheneu, 2004. 219p.

UNITED NATIONS ORGANIZATION - ONU. Food and Agriculture Organization/World Health - FAO/WHO. Recomendações dietéticas no relatório de uma joint WHO / FAO: consulta de especialistas em dieta, nutrição e prevenção de doenças crônicas. 2003. (WHO Technical Report Series 916).

VIDAL, G. M. et al. Avaliação das boas práticas em segurança alimentar de uma unidade de alimentação e nutrição de uma organização militar da cidade de Belém, PA. Revista Alim. Nutr. Araraquara, SP, v. 22, n. 2, p. 283-290, 2011. 